\title{
Rudin-like sets and hereditary families of compact sets
}

\author{
by \\ Étienne Matheron (Bordeaux) and Miroslav Zelený (Praha)
}

\begin{abstract}
We show that a comeager $\boldsymbol{\Pi}_{1}^{1}$ hereditary family of compact sets must have a dense $G_{\delta}$ subfamily which is also hereditary. Using this, we prove an "abstract" result which implies the existence of independent $\mathcal{M}_{0}$-sets, the meagerness of $\mathcal{U}_{0}$-sets with the property of Baire, and generalizations of some classical results of Mycielski. Finally, we also give some natural examples of true $F_{\sigma \delta}$ sets.
\end{abstract}

1. Introduction. A complex Borel measure on the circle group $\mathbb{T}$ is said to be a Rajchman measure if its Fourier transform vanishes at infinity. A set $A \subset \mathbb{T}$ is called a $\mathcal{U}_{0}$-set if $\mu(A)=0$ for all positive Rajchman measures $\mu$; otherwise, $A$ is an $\mathcal{M}_{0}$-set. These notions make sense in any nondiscrete (Hausdorff) locally compact abelian group $\mathbf{G}$.

It has been proved by Rudin $([\mathrm{R}])$ that there exist compact $\mathcal{M}_{0}$-sets which are linearly independent over the rationals; since then, independent $\mathcal{M}_{0}$-sets are called Rudin sets. Later on, Debs and Saint Raymond ([DStR], see also $[\mathrm{KL}]$ ) solved the longstanding "category problem" for $\mathcal{U}_{0}$-sets: any $\mathcal{U}_{0}$-set with the property of Baire is meager in $\mathbb{T}$; equivalently, any nonmeager $G_{\delta}$ set contains a compact $\mathcal{M}_{0^{-}}$-set. Both results assert that some family of "small" compact sets contains an $\mathcal{M}_{0^{-}}$set, but the formal analogy is in fact much stronger. Indeed, the families of small compact sets involved, namely the family of compact independent sets and the family of all compact subsets of some fixed nonmeager $G_{\delta}$ set, are both nonmeager in the hyperspace $\mathcal{K}(\mathbb{T})$, and both "determined by their finite members", which means that a compact set belongs to either family if and only if all its finite subsets do. In this paper, we stress this analogy by proving an abstract theorem that generalizes both results (Theorem 4.1). Roughly speaking, it says that if $\mathbf{B}$ is a family of probability measures on some Polish space $X$ which "looks like"

2000 Mathematics Subject Classification: 03E15, 28A05, 43A46.

The second author was supported by the grants MSM 0021620839 and GAČR 201/03/0931. One of the main results was obtained during the second author's stay at Université Bordeaux 1. The second author thanks Université Bordeaux 1 for the hospitality. 
the family of Rajchman measures, then any coanalytic nonmeager family of compact subsets of $X$ determined by its finite members must contain a set supporting some measure from $\mathbf{B}$.

The other main result of the paper (Theorem 2.2) is a general statement concerning hereditary families of compact sets. This result asserts that a comeager coanalytic family of compact sets which is hereditary for inclusion must contain a dense $G_{\delta}$ subfamily which is also hereditary. It follows (Corollary 2.10) that if $\mathcal{I}$ is a hereditary coanalytic nonmeager subfamily of $\mathcal{K}(X)$, where $X$ is some Polish space, then $\mathcal{I}$ contains a $G_{\delta}$ hereditary set $\mathcal{G}$ which is dense in $\mathcal{K}(U)$, for some nonempty open set $U \subset X$. This result is needed in the proof of Theorem 4.1. More importantly, we believe that it can be useful in various situations.

The paper is organized as follows. Section 2 is devoted to the proof of Theorem 2.2. In Section 3, we introduce the instrumental notion of nicely presented $F_{\sigma \delta}$ families of measures (those which "look like" the family of Rajchman measures) and give some examples. In Section 4, we prove Theorem 4.1 and give some consequences; these include Rudin's and Debs-Saint Raymond's results as well as generalizations of some classical results of Mycielski ([My]). Finally, using some of the previous ideas, we give some natural examples of true $F_{\sigma \delta}$ sets.

We now fix the notation and "background" material that will be used throughout the paper. All this material (and much more) can be found in $[\mathrm{K} 1]$.

If $X$ is a Polish space (that is, a separable, completely metrizable topological space), we denote by $\mathbf{M}(X)$ the family of all complex Borel measures on $X$, and by $\mathbf{P}(X)$ the family of all Borel probability measures on $X$. On $\mathbf{M}(X)$, and hence on $\mathbf{P}(X)$, two natural topologies are available. First, one can define the norm of a measure $\mu \in \mathbf{M}(X)$ by $\|\mu\|=|\mu|(X)$, where $|\mu|$ is the total variation of $\mu$, and consider the norm topology on $\mathbf{M}(X)$. A more useful topology is the so-called Prokhorov topology, the weak topology induced by the bounded continuous functions on $X$. Thus, a basic neighborhood of some measure $\mu_{0} \in \mathbf{M}(X)$ has the form

$$
\mathbf{U}=\left\{\mu \in \mathbf{M}(X) ;\left|\int f_{i} d \mu-\int f_{i} d \mu_{0}\right|<\varepsilon, i=1, \ldots, N\right\},
$$

where $f_{1}, \ldots, f_{N}$ are bounded continuous functions on $X$ and $\varepsilon$ is a positive number. It is well known that $\mathbf{P}(X)$ is Polish in the Prokhorov topology, while the norm topology is obviously not separable if $X$ is uncountable. In this paper, unless the norm is explicitly mentioned, all topological notions concerning $\mathbf{M}(X)$ or $\mathbf{P}(X)$ will refer to the Prokhorov topology. A subset $\mathbf{B}$ of $\mathbf{M}(X)$ or $\mathbf{P}(X)$ is said to be hereditary if it is downward closed under absolute continuity. For example, it is well known (and easy to prove) that the family of Rajchman measures on a nondiscrete LCA group $\mathbf{G}$ is hereditary. 
If $X$ is a Polish space, we denote by $\mathcal{K}(X)$ the space of all compact subsets of $X$, equipped with the Vietoris topology. Recall that this topology is generated by all sets of the form $\{K \in \mathcal{K}(X) ; K \cap U \neq \emptyset\}$ and $\{K \in$ $\mathcal{K}(X) ; K \subset U\}$, where $U$ is open in $X$, and that it is a Polish topology. A set $\mathcal{I} \subset \mathcal{K}(X)$ is said to be hereditary if it is downward closed under inclusion. For example, for any set $A \subset X$, the set $\mathcal{K}(A):=\{K \in \mathcal{K}(X) ; K \subset A\}$ is hereditary.

As a rule, we will use the now standard notation for Borel and projective classes; thus, for example, the symbols " $\boldsymbol{\Pi}_{3}^{0}$ ", " $\boldsymbol{\Sigma}_{1}^{1}$ ", and " $\boldsymbol{\Pi}_{1}^{1}$ " mean " $F_{\sigma \delta}$ ", "analytic", and "coanalytic" respectively. However, we also use the classical notations " $F_{\sigma}$ " and " $G_{\delta}$ " when they seem to be more suggestive. Finally, we denote by $\mathbb{N}$ the set of nonnegative integers, and by $\mathbb{N}^{*}$ the set of positive integers.

2. Comeager hereditary families of compact sets. In this section, $X$ is a Polish space. We shall say that a set $\mathcal{I} \subset \mathcal{K}(X)$ is big if $\mathcal{I}$ contains a dense $G_{\delta}$ hereditary set. This notion was instrumental in [M1], where the following question was raised.

\section{Problem 2.1. Is every comeager hereditary set $\mathcal{I} \subset \mathcal{K}(X)$ necessarily big?}

No definability assumption is made here on the set $\mathcal{I}$, but one can observe that a positive answer to 2.1 for $\boldsymbol{\Sigma}_{1}^{1}$ sets would yield a positive answer for all comeager hereditary sets $\mathcal{I}$. Indeed, if $\mathcal{I} \subset \mathcal{K}(X)$ is comeager, then $\mathcal{I}$ contains a dense $G_{\delta}$ set $\mathcal{H}$. Let $\mathcal{H}^{*}$ be the hereditary closure of $\mathcal{H}$, i.e.,

$$
\mathcal{H}^{*}=\{K \in \mathcal{K}(X) ; \exists L \in \mathcal{H}: K \subset L\} .
$$

The set $\mathcal{H}^{*}$ is hereditary, it is comeager since $\mathcal{H} \subset \mathcal{H}^{*}$, and if $\mathcal{I}$ is hereditary, then $\mathcal{H}^{*} \subset \mathcal{I}$. Finally, since the relation " $K \subset L$ " is closed in $\mathcal{K}(X) \times \mathcal{K}(X)$, the set $\mathcal{H}^{*}$ is also $\boldsymbol{\Sigma}_{1}^{1}$ in $\mathcal{K}(X)$. Thus, a positive answer to 2.1 for $\boldsymbol{\Sigma}_{1}^{1}$ sets implies a full positive answer.

It seems likely that, under some ad hoc set-theoretical assumption, the answer to this problem is in fact negative. Yet, we do have the following general positive result.

TheOREM 2.2. Every comeager hereditary $\Pi_{1}^{1}$ subset of $\mathcal{K}(X)$ is big.

For the proof of Theorem 2.2, the following family of closed subsets of $\mathcal{K}(X)$ will play a crucial role.

Definition 2.3. For each $n \in \mathbb{N}^{*}$, let us denote by $\mathcal{P}(n)$ the family of all (compact) subsets of $X$ containing at most $n$ points. We shall denote by $\mathfrak{H}$ the family of all closed sets $\mathcal{F} \subset \mathcal{K}(X)$ with the following property: $\mathcal{F} \cap \mathcal{P}(n)$ is nowhere dense in $\mathcal{P}(n)$ for each $n \in \mathbb{N}^{*}$. 
Since $\bigcup_{n} \mathcal{P}(n)$ is dense in $\mathcal{K}(X)$, it is clear that all sets in $\mathfrak{H}$ are nowhere dense. The following lemma gives a characterization of sets in $\mathfrak{H}$.

Lemma 2.4. For a closed set $\mathcal{F} \subset \mathcal{K}(X)$, the following are equivalent:

(1) $\mathcal{F} \in \mathfrak{H}$.

(2) There exists a dense open hereditary set $\mathcal{U} \subset \mathcal{K}(X)$ with $\mathcal{F} \cap \mathcal{U}=\emptyset$.

Proof. It is easy to check that if $\mathcal{A}$ is a dense hereditary subset of $\mathcal{K}(X)$, then $\mathcal{A} \cap \mathcal{P}(n)$ is dense in $\mathcal{P}(n)$ for all $n \in \mathbb{N}^{*}$. Thus, the complement of a dense open hereditary set belongs to $\mathfrak{H}$. Conversely, assume that $\mathcal{F} \in \mathfrak{H}$. To show that (2) is satisfied, we start with the following Claim.

Claim. For every open nonempty set $\mathcal{V} \subset \mathcal{K}(X)$ there exists an open hereditary set $\mathcal{W}$ such that $\mathcal{W} \cap \mathcal{V} \neq \emptyset$ and $\mathcal{W} \cap \mathcal{F}=\emptyset$.

Proof. We may assume that $\mathcal{V}$ has the form

$$
\mathcal{V}=\left\{K \in \mathcal{K}(X) ; K \subset U_{0}, K \cap U_{i} \neq \emptyset, i=1, \ldots, n\right\},
$$

where $U_{0} \subset X$ is open and the $U_{i}$ 's are pairwise disjoint open subsets of $U_{0}$. For each set $I=\left\{i_{1}, \ldots, i_{k}\right\} \subset\{1, \ldots, n\}$, let $\pi_{I}: U_{1} \times \cdots \times U_{n} \rightarrow \mathcal{K}(X)$ be the map defined by $\pi_{I}(x)=\left\{x_{i_{1}}, \ldots, x_{i_{k}}\right\}$. Since $\mathcal{F} \cap \mathcal{P}(k)$ is nowhere dense in $\mathcal{P}(k)$ for each $k \in\{1, \ldots, n\}$, all sets $O_{I}=\left\{x ; \pi_{I}(x) \notin \mathcal{F}\right\}$ are dense in $U_{1} \times \cdots \times U_{n}$. It follows that the intersection of all these (finitely many) open sets $O_{I}$ is nonempty; in other words, there exists a finite set $F \in \mathcal{V}$ with cardinality $n$ such that no nonempty subset of $F$ belongs to $\mathcal{F}$. Actually, since the isolated point $\emptyset$ is not in $\mathcal{F}$ either, no subset of $F$ belongs to $\mathcal{F}$. Therefore, if $W \supset F$ is a small enough open set, then the hereditary open set $\mathcal{W}=\mathcal{K}(W)$ is disjoint from $\mathcal{F}$. Indeed, otherwise one could find a sequence $\left(K_{i}\right) \subset \mathcal{K}(X)$, with $K_{i} \subset\left\{z \in X ; \varrho(z, F)<2^{-i}\right\}$ and $K_{i} \in \mathcal{F}$ for all $i$. Then some subsequence of $\left(K_{i}\right)$ converges to a set $K \subset F$; and since $\mathcal{F}$ is closed, $K$ is in $\mathcal{F}$, which is a contradiction.

Now, let $\mathcal{U}$ be the union of all hereditary open subsets of $\mathcal{K}(X)$ disjoint from the set $\mathcal{F}$. Then $\mathcal{U}$ is open, hereditary and $\mathcal{U} \cap \mathcal{F}=\emptyset$. Moreover, it follows from the Claim that $\mathcal{U}$ is also dense in $\mathcal{K}(X)$.

In what follows we shall denote by $\mathfrak{H}^{\text {ext }}$ the family of all sets $\mathcal{B} \subset \mathcal{K}(X)$ which can be covered by a countable family of sets from $\mathfrak{H}$ :

$$
\mathfrak{H}^{\text {ext }}=\left\{\mathcal{B} \subset \mathcal{K}(X) ; \exists\left(\mathcal{F}_{n}\right)_{n \in \mathbb{N}} \subset \mathfrak{H}: \mathcal{B} \subset \bigcup_{n=0}^{\infty} \mathcal{F}_{n}\right\} .
$$

With this notation, Lemma 2.4 immediately implies the following corollary.

Corollary 2.5. A set $\mathcal{I} \subset \mathcal{K}(X)$ is big if and only if $\mathcal{K}(X) \backslash \mathcal{I} \in \mathfrak{H}^{\text {ext }}$.

Proof. If $\mathcal{K}(X) \backslash \mathcal{I} \in \mathfrak{H}^{\text {ext }}$, let $\left(\mathcal{F}_{n}\right)$ be a sequence of sets from $\mathfrak{H}$ covering $\mathcal{K}(X) \backslash \mathcal{I}$, and pick dense open hereditary sets $\mathcal{U}_{n}$ such that $\mathcal{U}_{n} \cap \mathcal{F}_{n}=\emptyset$. 
Then $\mathcal{G}=\bigcap_{n} \mathcal{U}_{n}$ shows that $\mathcal{I}$ is big. The converse is proved in the same way, using the fact that any $G_{\delta}$ hereditary subset of $\mathcal{K}(X)$ is the intersection of a sequence of open hereditary sets. This well known fact can be seen as follows. Let $\widehat{X}$ be some metrizable compactification of $X$. Then $\mathcal{K}(X)$ is $G_{\delta}$ in $\mathcal{K}(\widehat{X})$, so a $G_{\delta}$ hereditary set $\mathcal{G} \subset \mathcal{K}(X)$ is $G_{\delta}$ in $\mathcal{K}(\widehat{X})$. Write $\mathcal{G}=\bigcap_{n} \widehat{\mathcal{V}}_{n}$, where the sets $\widehat{\mathcal{V}}_{n}$ are open in $\mathcal{K}(\widehat{X})$. Then the sets $\widehat{\mathcal{U}}_{n}=\left\{\widehat{K} \in \mathcal{K}(\widehat{X}) ; \mathcal{K}(\widehat{K}) \subset \widehat{\mathcal{V}}_{n}\right\}$ are open in $\mathcal{K}(\widehat{X})$ because $\widehat{X}$ is compact, and obviously hereditary. Thus, $\mathcal{U}_{n}=\mathcal{K}(X) \cap \widehat{\mathcal{U}}_{n}$ is open and hereditary in $\mathcal{K}(X)$ for all $n$, and $\mathcal{G}=\bigcap_{n} \mathcal{U}_{n}$.

By 2.5, proving Theorem 2.2 amounts to showing that if $\mathcal{I} \subset \mathcal{K}(X)$ is $\Pi_{1}^{1}$, comeager and hereditary, then $\mathcal{K}(X) \backslash \mathcal{I} \in \mathfrak{H}^{\text {ext }}$. Lemma 2.7 below implies this when $\mathcal{I}$ is assumed to be $\boldsymbol{\Sigma}_{2}^{0}$ instead of being merely $\boldsymbol{\Pi}_{1}^{1}$. Before proving this lemma, we collect some simple remarks on the space $\mathcal{K}(X)$.

We fix some compatible Polish metric $\varrho$ on $X$, and we associate to it the Hausdorff metric on $\mathcal{K}(X)$, which is Polish and compatible with the Vietoris topology. The open ball with center $K$ and radius $\varepsilon$ in $\mathcal{K}(X)$ will be denoted by $B(K, \varepsilon)$.

\section{LEMMA 2.6.}

(i) Let $\varepsilon>0, n \in \mathbb{N}^{*}, F \in \mathcal{K}(X)$, and $F^{*} \in \mathcal{P}(n)$ with $F^{*} \subset F$. If $T \in B(F, \varepsilon)$ then there exists $T^{*} \subset T$ such that $T^{*} \in \mathcal{P}(n) \cap B\left(F^{*}, \varepsilon\right)$.

(ii) Let $\varepsilon>0$ and $F, F^{*}, T^{*} \in \mathcal{K}(X)$. Assume that $F^{*} \subset F$ and $T^{*} \in$ $B\left(F^{*}, \varepsilon\right)$. Then there exists $T \in \mathcal{K}(X)$ such that $T^{*} \subset T$ and $T \in$ $B(F, \varepsilon)$.

Proof. (i) For every $x \in F^{*}$ one can find a point $y(x) \in T$ such that $\varrho(x, y(x))<\varepsilon$. Then the set $T^{*}=\left\{y(x) ; x \in F^{*}\right\}$ has the required properties.

(ii) Just put $T=T^{*} \cup F$.

Lemma 2.7. Let $\mathcal{L} \subset \mathcal{K}(X)$ be a $G_{\delta}$ set with $\mathcal{L} \notin \mathfrak{H}^{\text {ext }}$. Then the set

$$
\mathcal{T}=\left\{K \in \mathcal{K}(X) ; \exists K^{*} \in \mathcal{L}: K^{*} \subset K\right\}
$$

is not meager in $\mathcal{K}(X)$.

Proof. We will use the so-called Banach-Mazur game; let us recall its definition. Let $\mathcal{Y}$ be a metric space and $\mathcal{A} \subset \mathcal{Y}$. The Banach-Mazur game $G(\mathcal{A})=G(\mathcal{A}, \mathcal{Y})$ is defined as follows. There are two players, called $\mathbf{I}$ and II. They choose alternately nonempty open sets $\mathcal{U}_{1} \supset \mathcal{V}_{1} \supset \mathcal{U}_{2} \supset \mathcal{V}_{2} \supset \cdots$ :

$$
\begin{array}{llllll}
\text { I } & \mathcal{U}_{1} & \mathcal{U}_{2} & & \ldots \\
\text { II } & & \mathcal{V}_{1} & \mathcal{V}_{2} & \ldots
\end{array}
$$

Player II wins the game if $\mathcal{A} \cap \bigcap_{n=1}^{\infty} \mathcal{V}_{n}=\emptyset$; otherwise $\mathbf{I}$ wins. 
The main result concerning the Banach-Mazur game reads as follows (see $[\mathrm{K} 1])$ : the set $\mathcal{A}$ is meager in $\mathcal{Y}$ if and only if $\mathbf{I I}$ has a winning strategy in the game $G(\mathcal{A}, \mathcal{Y})$.

Now we turn to the proof of 2.7. By removing from $\mathcal{L}$ all its relatively open subsets which are in $\mathfrak{H}^{\text {ext }}$, we may and do assume that for every open

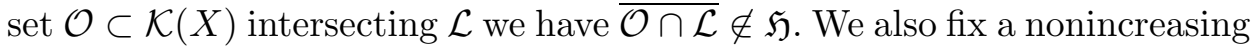
sequence $\left(\mathcal{L}_{n}\right)_{n \geq 1}$ of open sets such that $\mathcal{L}=\bigcap_{n=1}^{\infty} \mathcal{L}_{n}$.

We will show that the first player has a winning strategy in the BanachMazur game $G(\mathcal{T})=G(\mathcal{T}, \mathcal{K}(X))$. A fortiori, II has no winning strategy, hence the set $\mathcal{T}$ is not meager in $\mathcal{K}(X)$.

The second player will play nonempty open sets $\mathcal{V}_{n}$ 's and the first player will play balls $B\left(F_{n}, \varepsilon_{n}\right)$, with $\left.\varepsilon_{n} \in\right] 0,1 / n[$. Besides these balls, I will construct in the $n$th step an integer $j_{n} \in \mathbb{N}^{*}$ and a set $F_{n}^{*} \in \mathcal{P}\left(j_{n}\right)$ to obey the following conditions:

(a) $\overline{B\left(F_{n+1}, \varepsilon_{n+1}\right)} \subset B\left(F_{n}, \varepsilon_{n}\right)$;

(b) $F_{n}^{*} \subset F_{n}$;

(c) $\overline{B\left(F_{n+1}^{*}, \varepsilon_{n+1}\right)} \subset B\left(F_{n}^{*}, \varepsilon_{n}\right)$;

(d) $B\left(F_{n}^{*}, \varepsilon_{n}\right) \subset \mathcal{L}_{n}$;

(e) $\mathcal{P}\left(j_{n}\right) \cap B\left(F_{n}^{*}, \varepsilon_{n}\right) \subset \overline{\mathcal{L}}$.

The first step. Choose $K \in \mathcal{L}$ and $\tau>0$ such that $B(K, \tau) \subset \mathcal{L}_{1}$. We have $\overline{B(K, \tau / 2) \cap \mathcal{L}} \notin \mathfrak{H}$, so there exist an integer $j_{1} \in \mathbb{N}^{*}$, a set $F_{1}^{*} \in \mathcal{P}\left(j_{1}\right) \cap$ $B(K, \tau)$ and a positive number $\left.\varepsilon_{1} \in\right] 0,1\left[\right.$ such that $\mathcal{P}\left(j_{1}\right) \cap B\left(F_{1}^{*}, \varepsilon_{1}\right) \subset \overline{\mathcal{L}}$. Moreover, since $F_{1}^{*} \in B(K, \tau) \subset \mathcal{L}_{1}$, we may demand $B\left(F_{1}^{*}, \varepsilon_{1}\right) \subset \mathcal{L}_{1}$. Putting $F_{1}:=F_{1}^{*}$ finishes the description of I's first move.

The $(n+1)$ st step. Let $\mathcal{V}_{n} \subset B\left(F_{n}, \varepsilon_{n}\right)$ be the $n$th move of II. Choose $T \in \mathcal{K}(X)$ and $\delta>0$ such that $B(T, \delta) \subset \mathcal{V}_{n}$. To define the $(n+1)$ st move of I, we first find $T^{*} \in \mathcal{P}\left(j_{n}\right) \cap B\left(F_{n}^{*}, \varepsilon_{n}\right)$ such that $T^{*} \subset T$, which is possible by 2.6(i). Using (e), we find $K \in B\left(T^{*}, \delta\right) \cap B\left(F_{n}^{*}, \varepsilon_{n}\right) \cap \mathcal{L}$. Let $\tau>0$ be such that $B(K, \tau) \subset B\left(T^{*}, \delta\right) \cap B\left(F_{n}^{*}, \varepsilon_{n}\right) \cap \mathcal{L}_{n+1}$. We have $\overline{B(K, \tau / 2) \cap \mathcal{L}} \notin \mathfrak{H}$, so there exist $j_{n+1} \in \mathbb{N}^{*}, F_{n+1}^{*} \in \mathcal{P}\left(j_{n+1}\right) \cap B(K, \tau)$ and $\left.\varepsilon_{n+1} \in\right] 0,1 /(n+1)[$ such that $\mathcal{P}\left(j_{n+1}\right) \cap B\left(F_{n+1}^{*}, \varepsilon_{n+1}\right) \subset \overline{\mathcal{L}}$. Since $F_{n+1}^{*} \in B\left(F_{n}^{*}, \varepsilon_{n}\right) \cap \mathcal{L}_{n+1}$, we may demand $B\left(F_{n+1}^{*}, \varepsilon_{n+1}\right) \subset \mathcal{L}_{n+1}$ and $\overline{B\left(F_{n+1}^{*}, \varepsilon_{n+1}\right)} \subset B\left(F_{n}^{*}, \varepsilon_{n}\right)$. Since $F_{n+1}^{*} \in B\left(T^{*}, \delta\right)$, 2.6(ii) allows us to choose $F_{n+1} \in \mathcal{K}(X)$ such that $F_{n+1}^{*} \subset F_{n+1}$ and $F_{n+1} \in B(T, \delta)$; and taking $\varepsilon_{n+1}$ smaller if necessary, we also have $\overline{B\left(F_{n+1}, \varepsilon_{n+1}\right)} \subset B(T, \delta) \subset \mathcal{V}_{n}$. This finishes the description of I's strategy.

Let $B\left(F_{1}, \varepsilon_{1}\right), \mathcal{V}_{1}, B\left(F_{2}, \varepsilon_{2}\right), \mathcal{V}_{2}, \ldots$ be a sequence of moves in $G(\mathcal{T})$, where I follows the strategy described above. So there are also auxiliary sets $F_{1}^{*}, F_{2}^{*}, \ldots$ Since $\varepsilon_{n} \rightarrow 0$, it follows from (a) that there exists $F \in \mathcal{K}(X)$ such that $\{F\}=\bigcap_{n=1}^{\infty} \mathcal{V}_{n}$. According to (c), there exists $F^{*} \in \mathcal{K}(X)$ such 
that $\left\{F^{*}\right\}=\bigcap_{n=1}^{\infty} B\left(F_{n}^{*}, \varepsilon_{n}\right)$. Property (b) gives $F^{*} \subset F$, and properties (c) and (d) imply $F^{*} \in \bigcap_{n=1}^{\infty} \mathcal{L}_{n}=\mathcal{L}$. Thus we have $F \in \mathcal{T}$ and $\mathbf{I}$ has won.

The last ingredient needed in the proof of Theorem 2.2 is the following very useful covering theorem due to Solecki ([S]).

Theorem 2.8. Let $\mathcal{Y}$ be a Polish space, and let $\mathfrak{Q}$ be a family of closed subsets of $\mathcal{Y}$. If $\mathcal{B} \subset \mathcal{Y}$ is a $\boldsymbol{\Sigma}_{1}^{1}$ set which is not in $\mathfrak{Q}^{\text {ext }}$, then $\mathcal{B}$ contains a $G_{\delta}$ set which is not in $\mathfrak{Q}^{\text {ext }}$ either.

We can now give the

Proof of Theorem 2.2. Let $\mathcal{I} \subset \mathcal{K}(X)$ be $\Pi_{1}^{1}$, comeager, and hereditary. By 2.5, it is enough to show that $\mathcal{K}(X) \backslash \mathcal{I} \in \mathfrak{H}^{\text {ext }}$. So, towards a contradiction, assume that $\mathcal{K}(X) \backslash \mathcal{I} \notin \mathfrak{H}^{\text {ext }}$. The set $\mathcal{K}(X) \backslash \mathcal{I}$ is $\boldsymbol{\Sigma}_{1}^{1}$, hence according to Solecki's theorem, there is a $G_{\delta}$ set $\mathcal{L} \subset \mathcal{K}(X) \backslash \mathcal{I}$ such that $\mathcal{L} \notin \mathfrak{H}^{\text {ext }}$. Define

$$
\mathcal{T}=\left\{K \in \mathcal{K}(X) ; \exists K^{*} \in \mathcal{L}: K^{*} \subset K\right\} .
$$

Since $\mathcal{I}$ is hereditary, we have $\mathcal{T} \cap \mathcal{I}=\emptyset$. Using Lemma 2.7 we infer that $\mathcal{T}$ is not meager, a contradiction with comeagerness of $\mathcal{I}$.

REMARK. If, in addition to being comeager, the family $\mathcal{I}$ is also a $\sigma$ ideal, one may wonder if it must contain a dense $G_{\delta}(\sigma$-)ideal. By a result of Kechris $([\mathrm{K} 2])$, this is in fact not true; the family of $\mathcal{U}_{0}$-sets provides a counterexample.

As we said above, it seems likely that a positive answer to Problem 2.1 cannot be given in ZFC. On the other hand, the following result shows that a positive answer is nevertheless plausible.

Theorem 2.9. Assume that $\omega_{1}^{L[x]}<\omega_{1}$ for all $x \in \omega^{\omega}$. Then every comeager hereditary subset of $\mathcal{K}(X)$ is big.

Proof. As explained at the beginning of this section, it is enough to consider the case of a $\Sigma_{1}^{1}$ comeager hereditary set $\mathcal{I} \subset \mathcal{K}(X)$, and as before, it is enough to show that $\mathcal{K}(X) \backslash \mathcal{I} \in \mathfrak{H}^{\text {ext }}$. Now, it was proved by Solecki ([S, Corollary 2]) that, under the hypothesis " $\omega_{1}^{L[x]}<\omega_{1}$ for all $x \in \omega^{\omega}$ ", his covering Theorem 2.8 is valid for all $\boldsymbol{\Sigma}_{2}^{1}$ sets (and not just for $\boldsymbol{\Sigma}_{1}^{1}$ sets), provided the family $\mathfrak{Q}$ is $\boldsymbol{\Sigma}_{2}^{1}$ in the hyperspace $\mathcal{F}(\mathcal{K}(X))$ of all closed subsets of $\mathcal{K}(X)$; here, $\mathcal{F}(\mathcal{K}(X))$ is equipped with the Effros Borel structure (see [K1] for the definition). In the present situation, $\mathcal{K}(X) \backslash \mathcal{I}$ is $\Pi_{1}^{1}$ in $\mathcal{K}(X)$, and $\mathfrak{H}$ is Borel in $\mathcal{F}(\mathcal{K}(X))$. Thus, Solecki's theorem applies, and one can conclude the proof as above.

To conclude this section, we prove the following simple consequence of Theorem 2.2, which will be needed later. 
COROLlary 2.10. If $\mathcal{I} \subset \mathcal{K}(X)$ is $\boldsymbol{\Pi}_{1}^{1}$, nonmeager, and hereditary, then $\mathcal{I}$ contains a $G_{\delta}$ hereditary set $\mathcal{G}$ which is dense in $\mathcal{K}(U)$, for some nonempty open set $U \subset X$.

This follows at once from 2.2 and the next lemma.

LEMMA 2.11. If $\mathcal{I}$ is a nonmeager hereditary subset of $\mathcal{K}(X)$ with the property of Baire, then $\mathcal{I}$ is comeager in $\mathcal{K}(U)$, for some nonempty open set $U \subset X$.

Proof. Since $\mathcal{I}$ is nonmeager and has the property of Baire, it is comeager in some nonempty open set $\mathcal{U} \subset \mathcal{K}(X)$ of the form $\{K \in \mathcal{K}(X) ; K \subset U$, $K \cap U_{i} \neq \emptyset$ for $\left.i=1, \ldots, n\right\}$, where $U$ is an open subset of $X$ and $U_{1}, \ldots, U_{n}$ are open subsets of $U$. We show that $\mathcal{I}$ is comeager in $\mathcal{K}(U)$. Let

$$
\mathcal{F}=\mathcal{U} \backslash \mathcal{I} \quad \text { and } \quad \widetilde{\mathcal{F}}=\{K \in \mathcal{K}(X) ; \forall L \in \mathcal{U}: K \cup L \in \mathcal{F}\} .
$$

Since $\mathcal{I}$ is hereditary, $\mathcal{K}(U) \backslash \mathcal{I}$ is contained in $\widetilde{\mathcal{F}}$. Now, since $\mathcal{F}$ is meager and the map $(K, L) \mapsto K \cup L$ is continuous and open on the product space $\mathcal{K}(X) \times \mathcal{K}(X)$, the set $\{(K, L) \in \mathcal{K}(X) \times \mathcal{K}(X) ; K \cup L \in \mathcal{F}\}$ is meager in $\mathcal{K}(X) \times \mathcal{K}(X)$. By the Kuratowski-Ulam Theorem, it follows that $\widetilde{\mathcal{F}}$ is meager in $\mathcal{K}(X)$, and the proof is complete.

3. Nicely presented $\Pi_{3}^{0}$ sets. Let $B_{l^{\infty}}$ be the unit ball of the complex Banach space $l^{\infty}(\mathbb{N})=l^{1}(\mathbb{N})^{*}$, and denote by $w^{*}$ the $w^{*}$-topology on $B_{l \infty}$. Alternatively, $B_{l}$ is the infinite product $\overline{\mathbb{D}}^{\mathbb{N}}$, where $\overline{\mathbb{D}}$ is the closed unit disk in $\mathbb{C}$, and $w^{*}$ is simply the product topology. It is easy to check that the unit ball of $c_{0}$ is $\Pi_{3}^{0}$ in $\left(B_{l^{\infty}}, w^{*}\right)$. Moreover, by a classical result of Hahn (see [K1, 23.F] or $[\mathrm{Kö}])$, it is also $\boldsymbol{\Pi}_{3}^{0}$-complete. Actually, Hahn proved the following stronger result: if $B$ is any $\Pi_{3}^{0}$ set in a metrizable topological space $X$, then there exists a continuous map $\Phi: X \rightarrow\left(B_{l^{\infty}}, w^{*}\right)$ such that $\Phi^{-1}\left(c_{0}\right)=B$. Such a map $\Phi$ is called a continuous reduction.

For example, the family $\mathbf{R}$ of Rajchman probability measures on $\mathbb{T}$ is reduced to $c_{0}$ by the Fourier transform $\mathcal{F}: \mathbf{P}(\mathbb{T}) \rightarrow B_{l}$. Now, in that particular case, the reduction $\mathcal{F}$ has an additional property, which is in fact not innocent: it is also (\| \|, \| \|)-Lipschitz.

If $X$ is a Polish space, we shall say (with the example of $\mathbf{R}$ in mind) that a set $\mathbf{B} \subset \mathbf{P}(X)$ is nicely $\boldsymbol{\Pi}_{3}^{0}$ if it can be reduced to $c_{0}$ by a map $\Phi: \mathbf{P}(X) \rightarrow B_{l}$ which is both (Prokhorov, $w^{*}$ )-continuous and (\| $\left.\|\| \|,\right)$ Lipschitz. Such a set $\mathbf{B}$ has to be both $\boldsymbol{\Pi}_{3}^{0}$ in the Prokhorov topology and $\|$ ||-closed. The converse may seem to be plausible in view of Hahn's result, but it is in fact not true; see Examples 3 and 4 below.

EXAMPLE 1. If $X=\mathbf{G}$ is a second-countable, nondiscrete, locally compact abelian group, then the family $\mathbf{R}$ of Rajchman probability measures on $\mathbf{G}$ is nicely $\mathbf{\Pi}_{3}^{0}$. 
Proof. Let $\boldsymbol{\Gamma}$ be the dual group of $\mathbf{G}$, and let $\left(W_{n}\right)$ be an increasing sequence of open subsets of $\boldsymbol{\Gamma}$ with compact closures such that $\boldsymbol{\Gamma}=\bigcup_{n} W_{n}$. Then a measure $\mu$ on $\mathbf{G}$ is a Rajchman measure if and only if $\Phi_{n}(\mu)=$ $\sup \left\{|\widehat{\mu}(\gamma)| ; \gamma \in \bar{W}_{n+1} \backslash W_{n}\right\}$ tends to 0 as $n$ tends to infinity, so $\Phi=\left(\Phi_{n}\right)$ shows that $\mathbf{R}$ is nicely $\boldsymbol{\Pi}_{3}^{0}$.

EXAmple 2. If $X$ is a compact metric space, then the family $\mathbf{C}$ of all continuous Borel probability measures on $X$ is nicely $\Pi_{3}^{0}$. Moreover, $\mathbf{C}$ is also $\boldsymbol{\Pi}_{2}^{0}$.

Proof. Let us fix some countable basis for the topology of $X$, and some compatible metric on $X$. Let $\left(U_{n}, V_{n}\right)_{n \in \mathbb{N}}$ be an enumeration of all pairs of basic open sets $(U, V)$ such that $\bar{U} \subset V$, chosen in such a way that the diameter of $V_{n}$ tends to 0 as $n$ tends to infinity, and each pair $(U, V)$ with $V$ reduced to a single point is repeated infinitely many times. For each $n \in \mathbb{N}$, let $f_{n}$ be a continuous function on $X$ with support contained in $V_{n}$, such that $0 \leq f_{n} \leq 1$ and $f_{n} \equiv 1$ on $\bar{U}_{n}$. Then a measure $\mu \in \mathbf{P}(X)$ is continuous if and only if $\Phi_{n}(\mu)=\int f_{n} d \mu$ tends to 0 as $n$ tends to infinity, so $\Phi=\left(\Phi_{n}\right)$ shows that $\mathbf{C}$ is nicely $\boldsymbol{\Pi}_{3}^{0}$. That $\mathbf{C}$ is also $\boldsymbol{\Pi}_{2}^{0}$ can be seen as follows. For each $n \in \mathbb{N}$, let $\mathfrak{F}_{n}$ be a finite covering of $X$ by open sets of diameters less than $2^{-n}$. Then a measure $\mu \in \mathbf{P}(X)$ is continuous if and only if

$$
\forall k \in \mathbb{N} \exists n \in \mathbb{N} \forall V \in \mathfrak{F}_{n}: \mu(\bar{V})<2^{-k},
$$

which concludes the proof because the conditions " $\mu(\bar{V})<2^{-k}$ " are open.

Example 3. Let $X$ be a Polish space, let $A$ be any $\Pi_{3}^{0}$ subset of $X$, and let $\mathbf{B}=\mathbf{P}(A)$, the family of all Borel probability measures on $X$ concentrated on $A$ :

$$
\mathbf{P}(A)=\{\mu \in \mathbf{P}(X) ; \mu(X \backslash A)=0\} .
$$

Then $\mathbf{B}$ is both \|\| -closed and $\mathbf{\Pi}_{3}^{0}$. However, if $A$ is dense in $X$ and has the form $A=\bigcup_{n} K_{n}$, where $\left(K_{n}\right)$ is a sequence of pairwise disjoint (closed) nowhere dense perfect sets, then $\mathbf{P}(A)$ is not nicely $\mathbf{\Pi}_{3}^{0}$.

Proof. Write $A=\bigcap_{n} \bigcup_{p} F_{n p}$, where the $F_{n p}$ 's are closed subsets of $X$, all sequences $\left(F_{n p}\right), n \in \mathbb{N}$, are nondecreasing with respect to $p$, and $\left(\bigcup_{p} F_{n p}\right)$ is a nonincreasing sequence. Then a measure $\mu \in \mathbf{P}(X)$ is not concentrated on $A$ if and only if

$$
\exists k \in \mathbb{N} \exists n \in \mathbb{N} \forall p \in \mathbb{N}: \mu\left(F_{n p}\right)<1-2^{-k} .
$$

For each closed set $F \subset X$ and $\varepsilon>0$, the condition " $\mu(F)<\varepsilon$ " is open, so $\mathbf{P}(X) \backslash \mathbf{P}(A)$ is $\boldsymbol{\Sigma}_{3}^{0}$. The second part will follow from Theorem 4.1.

Example 4. Let $X$ be a Polish space, and let $\lambda \in \mathbf{P}(X)$. Then $\mathbf{B}_{\lambda}=$ $L^{1}(\lambda) \cap \mathbf{P}(X)$ is \|\| -closed and $\mathbf{\Pi}_{3}^{0}$. However, if $\lambda$ is a continuous measure, then $\mathbf{B}_{\lambda}$ is not nicely $\mathbf{\Pi}_{3}^{0}$. 
Proof. It is clear that $\mathbf{B}_{\lambda}$ is \|\| -closed, and it is also \|\| -separable. Let $D$ be a countable \|\| -dense set in $\mathbf{B}_{\lambda}$; then a probability measure $\mu$ is in $\mathbf{B}_{\lambda}$ if and only if

$$
\forall n \in \mathbb{N} \exists \nu \in D:\|\mu-\nu\| \leq 2^{-n} .
$$

Since the norm is lower semicontinuous on $\mathbf{M}(X)$, this shows that $\mathbf{B}_{\lambda}$ is $\mathbf{\Pi}_{3}^{0}$. It will follow from Theorem 4.1 that $\mathbf{B}_{\lambda}$ is not nicely $\boldsymbol{\Pi}_{3}^{0}$ if $\lambda$ is a continuous measure.

REMARK. In all the examples given above, the family $\mathbf{B}$ is more than just hereditary and \| \|-closed: it is also convex; in other words, the positive cone generated by $\mathbf{B}$ is a band of positive measures. For a detailed study of bands of measures and their polar $\sigma$-ideals, we refer to [D]. In particular, it is shown in $[\mathrm{D}]$ that any strongly convex Borel band has a " $c_{0}$-like" representation where the map $\Phi$ is moreover linear, and $c_{0}$ is replaced by $c_{\mathcal{F}}=\left\{\left(x_{n}\right) \in l^{\infty}\right.$; $\left.\lim _{\mathcal{F}} x_{n}=0\right\}$, for some Borel filter $\mathcal{F}$ on the integers. More general results on the complexity of families of the form $\mathbf{P}(A)$ can also be found in $[\mathrm{D}]$.

4. Finding big sets in large families of small sets. We now state and prove the abstract result mentioned in the introduction. Below, if $\mathbf{B}$ is a family of positive measures on $X$, a set $A \subset X$ is called an $\mathcal{I}_{\mathbf{B}}$-set if $\mu(A)=0$ for all measures $\mu \in \mathbf{B}$, and an $\mathcal{M}_{\mathbf{B}}$-set otherwise.

Theorem 4.1. Let $X$ be a Polish space, and let $\mathbf{B}$ be a hereditary subset of $\mathbf{P}(X)$. Assume that $\mathbf{B}$ is nicely $\mathbf{\Pi}_{3}^{0}$ and that $X$ is the support of some continuous measure in $\mathbf{B}$. Let also $\mathcal{I}$ be a nonmeager hereditary $\Pi_{1}^{1}$ subset of $\mathcal{K}(X)$. Then there exists a compact set $K \in \mathcal{M}_{\mathbf{B}}$ such that every finite subset of $K$ belongs to $\mathcal{I}$.

Since $\mathbf{B}$ is nicely $\boldsymbol{\Pi}_{3}^{0}$, we can choose once and for all a map

$$
\Phi=\left(\Phi_{n}\right): \mathbf{P}(X) \rightarrow B_{l^{\infty}},
$$

both $(\|\|,\|\|)$-Lipschitz and (Prokhorov, $\left.w^{*}\right)$-continuous, such that $\mathbf{B}=$ $\Phi^{-1}\left(c_{0}\right)$. We also fix some Polish compatible metric $d$ on $\mathbf{P}(X)$, and some (unspecified) compatible metric on $X$.

The proof of Theorem 4.1 is inspired by Körner's direct construction of Rudin sets (see [LP]). We shall need two lemmas.

Lemma 4.2. Let $F$ be a closed subset of $X$, let $\nu$ be a finite Borel positive measure on $F$, and let $\mathbf{U}$ be a neighborhood of $\nu$ in $\mathbf{M}(X)$. Then one can find $W_{1}, \ldots, W_{m}$, relatively open subsets of $F$ with pairwise disjoint closures, such that the following property holds: whenever a positive measure $\widetilde{\nu} \in$ $\mathbf{M}(F)$ satisfies $\|\widetilde{\nu}\| \leq\|\nu\|$ and $\widetilde{\nu}\left(W_{j}\right)=\nu\left(W_{j}\right)$ for $j=1, \ldots, m$ then $\widetilde{\nu} \in \mathbf{U}$. 
Proof. We assume $\nu \neq 0$, otherwise there is nothing to prove. We may also assume that $\mathbf{U}$ has the form

$$
\mathbf{U}=\left\{\widetilde{\nu} ;\left|\left\langle\widetilde{\nu}-\nu, f_{i}\right\rangle\right|<\varepsilon \text { for all } i \in I\right\},
$$

for some positive number $\varepsilon$ and some finite family $\left(f_{i}\right)_{i \in I}$ in the unit ball of $\mathcal{C}_{\mathrm{b}}(F)$. Let $K \subset F$ be a zero-dimensional compact set such that $\nu(K)>$ $\|\nu\|-\varepsilon / 4$. Then one can find relatively open sets $W_{1}, \ldots, W_{m} \subset F$ with pairwise disjoint closures such that $K \subset W_{1} \cup \cdots \cup W_{m}$ and, for each $j \in$ $\{1, \ldots, m\}$, the oscillation of all functions $f_{i}$ on $W_{j}$ is less than $\varepsilon / 4\|\nu\|$. Let $\widetilde{\nu}$ be any positive measure on $F$ such that $\|\widetilde{\nu}\| \leq\|\nu\|$ and $\widetilde{\nu}\left(W_{j}\right)=\nu\left(W_{j}\right)$ for $j=1, \ldots, m$. Putting $W=\bigcup_{j=1}^{m} W_{j}$, we have $\nu(F \backslash W), \widetilde{\nu}(F \backslash W)<\varepsilon / 4$, hence $\left|\int_{F \backslash W} f_{i} d \widetilde{\nu}-\int_{F \backslash W} f_{i} d \nu\right|<\varepsilon / 2$ for all $i \in I$. Moreover, if $i \in I$ then, taking any point $x_{j} \in W_{j}$, one gets

$$
\left|\int_{W_{j}} f_{i} d \widetilde{\nu}-\int_{W_{j}} f_{i} d \nu\right| \leq \int_{W_{j}}\left|f_{i}-f_{i}\left(x_{j}\right)\right| d \widetilde{\nu}+\int_{W_{j}}\left|f_{i}-f_{i}\left(x_{j}\right)\right| d \nu \leq \frac{\varepsilon}{2\|\nu\|} \nu\left(W_{j}\right)
$$

for all $j$; summing up, it follows that $\left|\int_{W} f_{i} d \widetilde{\nu}-\int_{W} f_{i} d \nu\right| \leq \varepsilon / 2$. Thus, we obtain $\left|\left\langle\widetilde{\nu}-\nu, f_{i}\right\rangle\right|<\varepsilon$ for all $i \in I$, and the proof is complete.

If $E, F$ are two closed subsets of $X$, we shall write $F \prec E$ if $F$ has the form $\bar{V}_{1} \cup \cdots \cup \bar{V}_{l}$, where the $V_{j}$ 's are nonempty relatively open subsets of $E$ with pairwise disjoint closures.

Lemma 4.3. Let $\mu$ be a continuous measure in $\mathbf{B}$, and let $\mathcal{U}$ be a dense hereditary open subset of $\mathcal{K}(\operatorname{supp}(\mu))$. Finally, let $U_{1}, \ldots, U_{p}$ be open subsets of $X$ at positive distance from each other. Then, for each $\varepsilon>0$, there exists a probability measure $\widetilde{\mu} \in \mathbf{B}$ with the following properties:

(1) $\|\Phi(\widetilde{\mu})-\Phi(\mu)\|_{\infty}<\varepsilon$;

(2) $d(\widetilde{\mu}, \mu)<\varepsilon$

(3) $\operatorname{supp}(\widetilde{\mu}) \prec \operatorname{supp}(\mu)$;

(4) if $\left(x_{1}, \ldots, x_{p}\right) \in U_{1} \times \cdots \times U_{p}$ and $x_{i} \in \operatorname{supp}(\widetilde{\mu})$ for all $i$, then $\left\{x_{1}, \ldots, x_{p}\right\} \in \mathcal{U}$.

Proof. Let $\alpha \in] 0,1[$ to be chosen later. Since $\mu$ is continuous, one can find $V_{1}, \ldots, V_{N}$, nonempty relatively open subsets of $\operatorname{supp}(\mu)$ with pairwise disjoint closures, such that $\mu\left(\bar{V}_{j}\right)<\alpha$ for all $j$ and $\mu\left(X \backslash \bigcup_{i} V_{i}\right)<\alpha$. We may also assume that no $V_{j}$ intersects more than one $U_{i}$, since the $V_{j}$ 's may be chosen to have arbitrarily small diameters and the $U_{i}$ 's are at positive distance from each other. Let $\mathcal{J}$ be the set of all $1-1$ sequences $\mathbf{j}=\left(j_{1}, \ldots, j_{p}\right)$ of length $p$, with terms in $\{1, \ldots, N\}$. We enumerate $\mathcal{J}$ as $\left\{\mathbf{j}^{1}, \ldots, \mathbf{j}^{M}\right\}$. For $\mathbf{j}=\left(j_{1}, \ldots, j_{p}\right) \in \mathcal{J}$, we put $V_{\mathbf{j}}=\left(V_{j_{1}} \cap U_{1}\right) \cup \cdots \cup\left(V_{j_{p}} \cap U_{p}\right)$.

We construct by induction probability measures $\widetilde{\mu}^{0}, \widetilde{\mu}^{1}, \ldots, \widetilde{\mu}^{M}$ in $\mathbf{B}$ and integers $n_{0}<n_{1}<\cdots<n_{M}$ with the following properties, whenever they make sense: 
(o) $\operatorname{supp}\left(\widetilde{\mu}^{0}\right) \subset \bigcup_{j} V_{j}$ and $\left\|\widetilde{\mu}^{0}-\mu\right\|<2 \alpha$;

(i) $\left|\Phi_{n}\left(\widetilde{\mu}^{k}\right)-\Phi_{n}\left(\widetilde{\mu}^{k-1}\right)\right|<2^{-k} \alpha$ if $n<n_{k-1}$;

(ii) $d\left(\widetilde{\mu}^{k}, \widetilde{\mu}^{k-1}\right)<2^{-k} \varepsilon$;

(iii) $\left\|\widetilde{\mu}^{k}-\widetilde{\mu}^{k-1}\right\|<2 p \alpha$;

(iv) $\left|\Phi_{n}\left(\widetilde{\mu}^{k}\right)\right|<\alpha$ if $n \geq n_{k}$;

(v) $\operatorname{supp}\left(\widetilde{\mu}^{k}\right) \prec \operatorname{supp}\left(\widetilde{\widetilde{\mu}}^{k-1}\right)$;

(vi) every compact subset of $\operatorname{supp}\left(\widetilde{\mu}^{k}\right) \cap V_{\mathbf{j}^{k}}$ is in $\mathcal{U}$.

For every $i \in\{1, \ldots, N\}$ we find a nonempty open set $P_{i}$ such that $\bar{P}_{i} \subset V_{i}$ and $\mu\left(X \backslash \bigcup_{i} \bar{P}_{i}\right)<\alpha$. Let

$$
\widetilde{\mu}^{0}=\frac{1}{\mu\left(\bigcup_{j} \bar{P}_{j}\right)} \mu_{\mid \bigcup_{j} \bar{P}_{j}} .
$$

Then, putting $P=\bigcup_{j} \bar{P}_{j}$ and $\beta=\mu(P)$, one gets

$$
\left\|\widetilde{\mu}^{0}-\mu\right\| \leq\left\|(1 / \beta-1) \mu_{\mid P}\right\|+\mu(X \backslash P)<(1-\beta)+\alpha<2 \alpha,
$$

so condition (o) is satisfied. Moreover, since $\widetilde{\mu}^{0} \in \mathbf{B}$, we can choose an integer $n_{0}$ such that $\left|\Phi_{n}\left(\widetilde{\mu}^{0}\right)\right|<\alpha$ if $n \geq n_{0}$.

Assume the construction has been carried out up to $k-1$. If $H:=$ $V_{\mathbf{j}^{k}} \cap \operatorname{supp}\left(\widetilde{\mu}^{k-1}\right)$ is empty, then we put $\widetilde{\mu}^{k}=\widetilde{\mu}^{k-1}$ and $n_{k}=1+n_{k-1}$. If $H \neq \emptyset$, then let $\nu$ be the restriction of $\widetilde{\mu}^{k-1}$ to $F:=\bar{H}$. Let also $\mathbf{U}$ be a neighborhood of $\nu$, to be specified later, and let $W_{1}, \ldots, W_{m}$ be the relatively open subsets of $F$ given by Lemma 4.2 . Since $F \prec \operatorname{supp}(\mu)$ by induction hypothesis $(\mathrm{v})$, the set $\mathcal{U} \cap \mathcal{K}(F)$ is dense in $\mathcal{K}(F)$. Since $\mathcal{U}$ is hereditary, it follows that one can find points $x_{1}, \ldots, x_{m}$ such that $x_{i} \in W_{i}$ for all $i$ and all subsets of $\left\{x_{1}, \ldots, x_{m}\right\}$ are in $\mathcal{U}$; and since $\mathcal{U}$ is open, one can therefore choose nonempty relatively open sets $O_{1}, \ldots, O_{m} \subset F$ such that $\bar{O}_{i} \subset W_{i}$ and every compact subset of $\bigcup_{i} \bar{O}_{i}$ is in $\mathcal{U}$. By Lemma 4.2, the measure

$$
\widetilde{\nu}=\sum_{i=1}^{m} \frac{\nu\left(W_{i}\right)}{\nu\left(\bar{O}_{i}\right)} \nu_{\mid \bar{O}_{i}}
$$

is in $\mathbf{U}$. If we now choose $\mathbf{U}$ small enough, then the measure $\widetilde{\mu}^{k}=\widetilde{\nu}+\widetilde{\mu}_{\mid X \backslash F}^{k-1}$ satisfies (i) and (ii). It also satisfies (v) and (vi), and (iii) as well because $\|\nu\|<p \alpha$. Moreover, $\widetilde{\mu}^{k}$ is in $\mathbf{B}$ because it is absolutely continuous with respect to $\widetilde{\mu}^{k-1}$, so one can choose $n_{k}>n_{k-1}$ satisfying (iv).

Let now $\widetilde{\mu}=\widetilde{\mu}^{M}$; we check that $\widetilde{\mu}$ has the required properties. By (v) and (ii), it is clear that $\operatorname{supp}(\widetilde{\mu}) \prec \operatorname{supp}(\mu)$ and $d(\widetilde{\mu}, \mu)<\varepsilon$. Moreover, if $\left(x_{1}, \ldots, x_{p}\right) \in \prod_{i=1}^{p} U_{i}$ and $x_{i} \in \operatorname{supp}(\widetilde{\mu}), i=1, \ldots, p$, then there is some index $k \in\{1, \ldots, M\}$ such that $\left\{x_{1}, \ldots, x_{p}\right\} \subset V_{\mathbf{j}^{k}}$, because $\operatorname{supp}(\widetilde{\mu}) \subset \bigcup_{j} V_{j}$ (by (o)) and no $V_{j}$ intersects more than one $U_{i}$. Since $\operatorname{supp}(\widetilde{\mu}) \subset \operatorname{supp}\left(\widetilde{\mu}^{k}\right)$, it follows from (vi) that $\left\{x_{1}, \ldots, x_{p}\right\} \in \mathcal{U}$. Finally, let $n \in \mathbb{N}$, and let $k$ be 
the greatest integer in $\{-1,0, \ldots, M\}$ such that $n_{k} \leq n$ (we put $n_{-1}=0$ ). If $k \geq 0$, then, denoting by $C$ the Lipschitz constant of $\Phi$, we get

$$
\begin{aligned}
\left|\Phi_{n}(\widetilde{\mu})-\Phi_{n}(\mu)\right| & \leq C\left\|\widetilde{\mu}^{0}-\mu\right\|+\left|\Phi_{n}\left(\widetilde{\mu}^{k}\right)-\Phi_{n}\left(\widetilde{\mu}^{0}\right)\right|+\left|\Phi_{n}(\widetilde{\mu})-\Phi_{n}\left(\widetilde{\mu}^{k}\right)\right| \\
& \leq 2 C \alpha+2 \alpha+\left|\Phi_{n}(\widetilde{\mu})-\Phi_{n}\left(\widetilde{\mu}^{k}\right)\right| \\
& \leq 2 \alpha(C+1)+C\left\|\widetilde{\mu}^{k+1}-\widetilde{\mu}^{k}\right\|+\sum_{k^{\prime}>k+1}\left|\Phi_{n}\left(\widetilde{\mu}^{k^{\prime}}\right)-\Phi_{n}\left(\widetilde{\mu}^{k^{\prime}-1}\right)\right| \\
& \leq 2 \alpha(C+1)+2 C p \alpha+\alpha \sum_{k^{\prime}>k+1} 2^{-k^{\prime}} .
\end{aligned}
$$

If $k=-1$, then $n<n_{0}$ and we simply get

$$
\begin{aligned}
\left|\Phi_{n}(\widetilde{\mu})-\Phi_{n}(\mu)\right| & \leq C\left\|\widetilde{\mu}^{0}-\mu\right\|+\left|\Phi_{n}(\widetilde{\mu})-\Phi_{n}\left(\widetilde{\mu}^{0}\right)\right| \\
& \leq 2 C \alpha+\sum_{k=1}^{M}\left|\Phi_{n}\left(\widetilde{\mu}^{k}\right)-\Phi_{n}\left(\widetilde{\mu}^{k-1}\right)\right| \leq 2 C \alpha+\alpha .
\end{aligned}
$$

It follows that $\|\Phi(\widetilde{\mu})-\Phi(\mu)\|_{\infty}<\varepsilon$ if $\alpha$ is chosen small enough.

Proof of Theorem 4.1. By 2.10, the family $\mathcal{I}$ contains a $G_{\delta}$ hereditary set $\mathcal{G}$ which is dense in $\mathcal{K}(U)$, for some nonempty open set $U \subset X$; and replacing $X$ by $U$, we may in fact assume that $\mathcal{G}$ is dense in $\mathcal{K}(X)$. Let $\left(\mathcal{U}^{n}\right)$ be a nonincreasing sequence of open hereditary subsets of $\mathcal{K}(X)$ such that $\mathcal{G}=\bigcap_{n} \mathcal{U}^{n}$ (see the proof of 2.5). Let us also fix some countable basis for the topology of $X$, and let $\left(\mathbf{U}^{n}\right)$ be an enumeration of all finite sequences $\left(U_{1}, \ldots, U_{p}\right)$ of basic open subsets of $E$ whose closures are at positive distance from each other, where each sequence is repeated infinitely many times.

If $F \subset X$ is any closed set such that $F \prec X$, then $\mathcal{U}^{n} \cap \mathcal{K}(F)$ is dense in $\mathcal{K}(F)$ for all $n \in \mathbb{N}$. By Lemma 4.3 , one can therefore construct a sequence $\left(\mu^{n}\right) \subset \mathbf{B}$ such that the following properties hold:

(1) $d\left(\mu^{n+1}, \mu^{n}\right)<2^{-n}$;

(2) $\left\|\Phi\left(\mu^{n+1}\right)-\Phi\left(\mu^{n}\right)\right\|_{\infty}<2^{-n}$;

(3) $\operatorname{supp}\left(\mu^{n+1}\right) \prec \operatorname{supp}\left(\mu^{n}\right)$;

(4) write $\mathbf{U}^{n}=\left(U_{1}, \ldots, U_{p}\right)$; if $\left(x_{1}, \ldots, x_{p}\right) \in U_{1} \times \cdots \times U_{p}$ and $x_{i} \in$ $\operatorname{supp}\left(\mu^{n}\right)$ for all $i \in\{1, \ldots, p\}$, then $\left\{x_{1}, \ldots, x_{p}\right\} \in \mathcal{U}^{n}$.

By (1), the sequence $\left(\mu^{n}\right)$ has a limit $\mu^{\infty} \in \mathbf{P}(X)$. Since $\Phi$ is $w^{*}$-continuous, $\Phi\left(\mu^{\infty}\right)$ is the $w^{*}$-limit of the sequence $\left(\Phi\left(\mu^{n}\right)\right)$. By $(2)$, it follows that $\Phi\left(\mu^{\infty}\right) \in c_{0}$, so $\mu^{\infty} \in$ B. Moreover, conditions (3) and (4) ensure that any finite subset of $\operatorname{supp}\left(\mu^{\infty}\right)$ is in $\mathcal{G}$, hence in $\mathcal{I}$. Thus any compact set $K \subset \operatorname{supp}\left(\mu^{\infty}\right)$ with positive $\mu^{\infty}$-measure satisfies the conclusion of the theorem.

REmARKS. (1) As we have already said, the examples given above are all convex families of measures. Yet, it may be worth pointing out that the preceding proof does not need any convexity assumption on the family $\mathbf{B}$. 
(2) In general, one cannot hope to get a set $K \in \mathcal{M}_{\mathbf{B}} \cap \mathcal{I}$. For example, the family of compact $\mathcal{U}_{0}$-sets is $\Pi_{1}^{1}$ comeager in $\mathcal{K}(\mathbb{T})$, and surely does not contain any $\mathcal{M}_{0}$-set.

(3) The proof of Theorem 4.1 actually showed that, starting from any continuous measure $\mu \in \mathbf{B}$ with support $X$, one can construct a measure $\mu^{\infty} \in \mathbf{B}$ such that all finite subsets of $\operatorname{supp}\left(\mu^{\infty}\right)$ are in $\mathcal{I}$, with $\| \Phi\left(\mu^{\infty}\right)-$ $\Phi(\mu) \|_{\infty}$ arbitrarily small. This remark will be used in the proof of Corollary 4.11 below.

(4) It is easily seen that Theorem 4.1 is still true, with exactly the same proof, if one extends the class of nicely presented $\Pi_{3}^{0}$ sets by allowing the reduction $\Phi: \mathbf{P}(X) \rightarrow B_{l \infty}$ to be (\|\|, \| \|)-uniformly continuous rather than $(\|\|,\|\|)$-Lipschitz. On the other hand, the proof does not work any more if the map $\Phi$ is only assumed to be $(\|\|,\|\|)$-continuous, and we do not know if Theorem 4.1 is still true in this more general setting.

We now list some consequences of Theorem 4.1.

COROLlary 4.4. If $\mathbf{G}$ is a nondiscrete, second countable LCA group, then any $\mathcal{M}_{0}$-set in $\mathbf{G}$ contains a compact $\mathcal{M}_{0}$-set $K$ such that all finite subsets of $K$ are Kronecker sets. In particular, $K$ is an independent $\mathcal{M}_{0}$-set.

Proof. Recall that a compact set $C \subset \mathbf{G}$ is called a Kronecker set if $C$ is totally disconnected and every continuous function $f: C \rightarrow \mathbb{T}_{q}$ can be uniformly approximated by characters of $\mathbf{G}$, where $q=q(G)$ is the topological order of $\mathbf{G}, \mathbb{T}_{q}=\mathbb{T}$ if $q=\infty$ and $\mathbb{T}_{q}=\left\{z \in \mathbb{T} ; z^{q}=1\right\}$ if $q<\infty$. It is well known that a finite set is Kronecker if and only if it is independent and all its elements have order $q(\mathbf{G})$. Moreover, the family of Kronecker subsets of $\mathbf{G}$ is hereditary and $G_{\delta}$ in $\mathcal{K}(\mathbf{G})$, and it is not difficult to show that if $E \subset \mathbf{G}$ is the support of a Rajchman measure, then the Kronecker sets contained in $E$ are dense in $\mathcal{K}(E)$ (see [M1]). Since Rajchman measures are continuous and every $\mathcal{M}_{0}$-set contains such a set $E$, the result now follows directly from 4.1 .

COROllary 4.5. If $\mathbf{G}$ is a nondiscrete, second countable LCA group, and if $E \subset \mathbf{G}$ is the support of a Rajchman measure, then every $\mathcal{U}_{0}$ subset of $E$ with the property of Baire is meager in $E$.

Proof. If $A \subset E$ is nonmeager and has the property of Baire, it contains a $G_{\delta}$ set $H$ which is dense in some nonempty open subset of $E$. Then $\widetilde{E}=\bar{H}$ is the support of a Rajchman measure, and $\mathcal{I}=\mathcal{K}(H)$ is $G_{\delta}$, hereditary, and dense in $\mathcal{K}(\widetilde{E})$. By Theorem 4.1, it follows that $H$ is an $\mathcal{M}_{0}$-set, hence $A$ is an $\mathcal{M}_{0}$-set as well.

The next corollary is a generalization of a well known Mycielski-type result (see $[\mathrm{My}]$ ). 
COROLlary 4.6. Let $\mathbf{G}$ be a nondiscrete, second countable LCA group, and let $\left(R_{n}\right)$ be a sequence of finitary relations on $\mathbf{G}$; thus $R_{n}$ is a subset of $\mathbf{G}^{k_{n}}$, for some positive integer $k_{n}$. Assume that for each $n$, the relation $R_{n}$ is meager in $\mathbf{G}^{k_{n}}$. Then there exists an $\mathcal{M}_{0}$-set $K \subset \mathbf{G}$ with the following property: if $n \in \mathbb{N}$ and $x_{1}, \ldots, x_{k_{n}}$ are pairwise distinct points of $K$, then $\neg R_{n}\left(x_{1}, \ldots, x_{k_{n}}\right)$.

Proof. We may obviously assume that all relations $R_{n}$ are $F_{\sigma}$. Let $\mathcal{I}$ be the family of all compact subsets of $\mathbf{G}$ with the above property. It is easy to check that $\mathcal{I}$ is a $G_{\delta}$ subset of $\mathcal{K}(\mathbf{G})$, obviously hereditary. Since $\mathcal{I}$ is determined by its finite members, it is enough, by Theorem 4.1, to show that $\mathcal{I}$ is dense in $\mathcal{K}(\mathbf{G})$. Moreover, by the Baire Category Theorem, we may assume that there is just one meager $F_{\sigma}$ relation $R \subset \mathbf{G}^{k}$. Let $\mathbb{N}^{(k)}$ be the family of all finite subsets of $\mathbb{N}$ with cardinality $k$. For each set $I=\left\{i_{1}, \ldots, i_{k}\right\} \in \mathbb{N}^{(k)}$, let $\pi_{I}: \mathbf{G}^{\mathbb{N}} \rightarrow \mathbf{G}^{k}$ be the "canonical projection" $\left(\pi_{I}(x)=\left(x_{i_{1}}, \ldots, x_{i_{k}}\right)\right)$. By the Kuratowski-Ulam Theorem, all sets $B_{I}=$ $\left\{\left(x_{n}\right) \in \mathbf{G}^{\mathbb{N}} ; \neg R\left(\pi_{I}(x)\right)\right\}$ are comeager in the Polish space $\mathbf{G}^{\mathbb{N}}$, so $B=$ $\bigcap_{I \in \mathbb{N}^{(k)}} B_{I}$ is also comeager. It follows that each finite subset of $\mathbf{G}$ can be approximated by a (finite) set in $\mathcal{I}$, hence $\mathcal{I}$ is dense in $\mathcal{K}(\mathbf{G})$.

Corollary 4.7. Given any dense $G_{\delta}$ set $G \subset \mathbb{R}$ containing 0 , there exists an algebraically independent $\mathcal{M}_{0}$-set $K \subset \mathbb{R}$ such that the subgroup generated by $K$ is contained in $G$.

Proof. Let $\left(f_{n}\right)$ be an enumeration of all nonconstant polynomial functions in finitely many real variables with rational coefficients, $f_{n}: \mathbb{R}^{k_{n}}$ $\rightarrow \mathbb{R}$. Since the $f_{n}$ 's are real-analytic, their level sets are nowhere dense, in other words the relations " $f_{n}\left(x_{1}, \ldots, x_{k_{n}}\right)=0$ " are meager. Moreover, if $\left(n_{1}, \ldots, n_{p}\right)$ is a finite sequence of nonzero integers, the relation " $\sum_{i=1}^{p} n_{i} x_{i}$ $\notin G$ " is meager in $\mathbb{R}^{p}$ by the Kuratowski-Ulam Theorem, because all its sections along the $x_{1}$-axis are translates of the meager set $\left(1 / n_{1}\right)(\mathbb{R} \backslash G)$. Thus, the result follows from Corollary 4.6.

COROLlary 4.8. There exists an $\mathcal{M}_{0}$-set $K \subset \mathbb{R}^{2}$ that meets each line in at most 2 points.

Proof. Let $R$ be the relation defined on $\mathbb{R}^{6}=\mathbb{R}^{2} \times \mathbb{R}^{2} \times \mathbb{R}^{2}$ by

$$
R(a, b, c) \Leftrightarrow a, b, c \text { lie on the same line. }
$$

This relation is obviously nowhere dense, so the result follows from Corollary 4.6 .

Corollary 4.9. Let $X$ be a Polish space and let $A$ be a dense subset of $X$ of the form $A=\bigcup_{n} K_{n}$, where $\left(K_{n}\right)$ is a sequence of pairwise disjoint (closed) nowhere dense perfect sets. Then $\mathbf{B}=\mathbf{P}(A)$ is not nicely $\mathbf{\Pi}_{3}^{0}$. 
Proof. The hypotheses on $A$ imply that $\mathbf{B}$ contains a continuous measure with support $X$. But $G=X \backslash A$ is a dense $G_{\delta}$ subset of $X$ and no measure in B is supported on $G$. By Theorem 4.1 applied to $\mathcal{I}=\mathcal{K}(G)$, the family B cannot be nicely $\boldsymbol{\Pi}_{3}^{0}$.

Corollary 4.10. Let $X$ be a Polish space, and let $\lambda$ be a continuous Borel probability measure on $X$. Then $\mathbf{B}_{\lambda}=L^{1}(\lambda) \cap \mathbf{P}(X)$ is not nicely $\boldsymbol{\Pi}_{3}^{0}$.

Proof. Let $E=\operatorname{supp}(\lambda)$, and let $G \subset E$ be a dense $G_{\delta}$ set such that $\lambda(G)=0$; such a set $G$ exists because $E$ has no isolated points. If $\mathbf{B}_{\lambda}$ were nicely $\boldsymbol{\Pi}_{3}^{0}$, then one should have $\lambda(G)>0$ by Theorem 4.1 applied to $\mathcal{I}=\mathcal{K}(G)$.

This last corollary can be strengthened as follows.

Corollary 4.11. Let $X$ be a compact metric space, and let $\mathbf{B} \subset \mathbf{P}(X)$ be hereditary and nicely $\mathbf{\Pi}_{3}^{0}$. If $\mathbf{B}$ contains a continuous measure, then the $\sigma$-ideal $\mathcal{I}_{\mathbf{B}}$ is not thin, in other words there exists an uncountable family of pairwise disjoint $\mathcal{M}_{\mathbf{B}}$-sets. In particular, $\mathbf{B}$ is not \|\| -separable.

Proof. Let $\mathbf{B}_{\mathrm{c}}$ be the family of all continuous measures in $\mathbf{B}$. Then $\mathbf{B}_{\mathrm{c}}$ is still hereditary. Moreover, $\mathbf{B}_{\mathrm{c}}$ is also nicely $\mathbf{\Pi}_{3}^{0}$. Indeed, the family $\mathbf{C}$ of continuous probability measures on $X$ is nicely $\boldsymbol{\Pi}_{3}^{0}$ (Example 2 above), and one checks immediately (using a product map $\Phi=\left(\Phi^{1}, \Phi^{2}\right): \mathbf{P}(X) \rightarrow$ $\left.l^{\infty} \times l^{\infty} \simeq l^{\infty}\right)$ that the intersection of two nicely $\boldsymbol{\Pi}_{3}^{0}$ sets is again nicely $\boldsymbol{\Pi}_{3}^{0}$. Let $\Phi: \mathbf{P}(X) \rightarrow B_{l^{\infty}}$ be (Prokhorov, $w^{*}$ )-continuous and (\| \|, \| \|)-Lipschitz with $\Phi^{-1}\left(c_{0}\right)=\mathbf{B}_{\mathrm{c}}$. It is enough to prove the following

FACT. If $\mu \in \mathbf{B}_{\mathrm{c}}$ and $\varepsilon>0$ are given, then there exist two measures $\mu_{0}, \mu_{1} \in \mathbf{B}_{\mathrm{c}}$ with pairwise disjoint supports contained in $\operatorname{supp}(\mu)$ and such that $\left\|\Phi\left(\mu_{i}\right)-\Phi(\mu)\right\|_{\infty}<\varepsilon, i=0,1$.

Indeed, once this is done, one can construct a Cantor scheme $\left(\mu_{s}\right)_{s \in 2<\omega}$ $\subset \mathbf{B}_{\mathrm{c}}$ with $\left\|\mu_{s}-\mu_{t}\right\|<2^{-|s|}$ and $\operatorname{supp}\left(\mu_{t}\right) \subset \operatorname{supp}\left(\mu_{s}\right)$ if $s \preceq t$, while $\operatorname{supp}\left(\mu_{s}\right) \cap \operatorname{supp}\left(\mu_{t}\right)=\emptyset$ if $s$ and $t$ are incomparable. Since $\mathbf{B}_{\mathrm{c}}$ is norm closed in $\mathbf{P}(X)$, this gives an uncountable family $\left(\mu_{\alpha}\right)_{\alpha \in 2^{\omega}}$ of measures in $\mathbf{B}_{\mathrm{c}}$ with pairwise disjoint supports.

Let us now prove the above Fact. Let $E$ be the support of $\mu$, and let $G_{0} \subset E$ be a dense $G_{\delta}$ set in $E$ with empty interior in $E$; such a set $G_{0}$ exists because $E$ is perfect. By the proof of Theorem 4.1 applied to $\mathbf{B}_{\mathrm{c}} \cap \mathbf{P}(E)$ and $\mathcal{I}_{0}=\mathcal{K}\left(G_{0}\right) \subset \mathcal{K}(E)$ (see Remark (3) following that proof), one can find a measure $\mu_{0} \in \mathbf{B}_{\mathrm{c}}$ with compact support contained in $G_{0}$, such that $\left\|\Phi\left(\mu_{0}\right)-\Phi(\mu)\right\|_{\infty}<\varepsilon$. Then $\operatorname{supp}\left(\mu_{0}\right)$ is nowhere dense in $E$, so we can get the measure $\mu_{1}$ by applying (the proof of) 4.1 to $\mathcal{I}_{1}=\mathcal{K}\left(G_{1}\right)$, where $G_{1}=$ $E \backslash \operatorname{supp}\left(\mu_{0}\right) \neq \emptyset$. This concludes the proof of the Fact, and Corollary 4.11 follows. 
If $\mathbf{B}$ is the family of continuous measures on some perfect compact metric space, Theorem 4.1 can of course be greatly improved: by the Baire Category Theorem, any dense $G_{\delta}$ subset of $\mathcal{K}(X)$ contains a perfect set, hence an $\mathcal{M}_{\mathbf{B}}$-set. A similar statement holds true for any $\boldsymbol{\Pi}_{2}^{0}$ family $\mathbf{B}$. The proof is also a simple application of the Baire Category Theorem.

Proposition 4.12. Let $X$ be a Polish space, and let $\mathbf{B} \subset \mathbf{P}(X)$ be hereditary and $\mathbf{\Pi}_{2}^{0}$. Assume that $X$ is the support of some measure in $\mathbf{B}$. Then every nonmeager hereditary $\Pi_{1}^{1}$ subset of $\mathcal{K}(X)$ contains an $\mathcal{M}_{\mathbf{B}}$-set.

Proof. Since $X$ is the support of some measure in $\mathbf{B}$ and $\mathbf{B}$ is hereditary, it follows easily from Lemma 4.2 that $\mathbf{B}$ is dense in $\mathbf{P}(X)$. It also follows from 4.2 that if $\mathcal{U}$ is a dense hereditary open subset of $\mathcal{K}(X)$ and $\varepsilon \in] 0,1[$, then the family

$$
\mathbf{P}_{\mathcal{U} \varepsilon}=\{\mu \in \mathbf{P}(X) ; \mu(V)>1-\varepsilon \text { for some } V \subset X \text { open with } \mathcal{K}(V) \subset \mathcal{U}\}
$$

is dense in $\mathbf{P}(X)$. Moreover, $\mathbf{P}_{\mathcal{U} \varepsilon}$ is also open in $\mathbf{P}(X)$. Now, let $\mathcal{I}$ be a nonmeager hereditary $\boldsymbol{\Pi}_{1}^{1}$ subset of $\mathcal{K}(X)$. By Corollary 2.10, we may in fact assume that $\mathcal{I}$ contains a dense $G_{\delta}$ hereditary set $\mathcal{G}$. Let $\left(\mathcal{U}_{n}\right)$ be a sequence of dense hereditary open sets such that $\mathcal{G}=\bigcap_{n} \mathcal{U}_{n}$, and let also $\left(\varepsilon_{n}\right)$ be a sequence of positive numbers such that $\sum_{n=0}^{\infty} \varepsilon_{n}<1$. Since $\mathbf{B}$ is $\boldsymbol{\Pi}_{2}^{0}$ and dense in $\mathbf{P}(X)$, it follows from the Baire Category Theorem that $\bigcap_{n} \mathbf{P}_{\mathcal{U}_{n} \varepsilon_{n}}$ intersects $\mathbf{B}$; in other words, one can find a measure $\mu \in \mathbf{B}$ and a sequence $\left(V_{n}\right)$ of open subsets of $X$ such that $\mu\left(V_{n}\right)>1-\varepsilon_{n}$ and $\mathcal{K}\left(V_{n}\right) \subset \mathcal{U}_{n}$ for all $n \in \mathbb{N}$. Then $G=\bigcap_{n} V_{n}$ has positive $\mu$-measure, and all compact subsets of $G$ are in $\mathcal{G}$, hence in $\mathcal{I}$. This concludes the proof.

5. Some examples of true $\Pi_{3}^{0}$ sets. To conclude this paper, we give some examples of natural true $\Pi_{3}^{0}$ sets.

First, we give a short proof (and a slight extension) of a recent result due to Balcerzak and Darji ([BD]). Below, if $X$ is a Polish space and if $\mathcal{I}$ is a hereditary subset of $\mathcal{K}(X)$, we say that a compact set $E \subset X$ is $\mathcal{I}$-perfect if all sets in $\mathcal{I} \cap \mathcal{K}(E)$ are nowhere dense in $E$; equivalently, if $\bar{V} \cap E \notin \mathcal{I}$ for all open sets $V$ such that $V \cap E \neq \emptyset$. We denote by $\mathcal{I}^{\text {perf }}$ the family of $\mathcal{I}$-perfect sets.

Proposition 5.1. Let $X$ be a Polish space, and let $\mathcal{I}$ be a hereditary subset of $\mathcal{K}(X)$ with the Baire property. Assume that $\mathcal{I}$ is nonmeager, and that $\mathcal{I}^{\text {perf }}$ is dense in $\mathcal{K}(X)$. Then $\mathcal{I}^{\text {perf }}$ is not $\boldsymbol{\Sigma}_{3}^{0}$ in $\mathcal{K}(X)$.

Proof. We may assume that $\mathcal{I}^{\text {perf }}$ is Borel, otherwise there is nothing to prove. By Lemma 2.11, we may assume that $\mathcal{I}$ is comeager in $\mathcal{K}(X)$. Since $\mathcal{I}^{\text {perf }}$ is dense and disjoint from $\mathcal{I}$ (apart from the empty set), it follows from the Baire Category Theorem that if $V$ is any nonempty open subset of $X$, then $\mathcal{I}^{\text {perf }} \cap \mathcal{K}(V)$ cannot be $\Pi_{2}^{0}$ in $\mathcal{K}(V)$. Since $\mathcal{I}^{\text {perf }}$ is assumed to be 
Borel, it follows from Wadge's Theorem (see [K1, 22.11]) that $\mathcal{I}^{\text {perf }} \cap \mathcal{K}(V)$ is $\boldsymbol{\Sigma}_{2}^{0}$-hard, for each nonempty open set $V \subset X$; in other words, given any $\boldsymbol{\Sigma}_{2}^{0}$ set $A$ in some zero-dimensional Polish space $Z$, there exists a continuous map $\Psi: Z \rightarrow \mathcal{K}(V)$ such that $A=\Psi^{-1}\left(\mathcal{I}^{\text {perf }}\right)$. We use this to show that $\mathcal{I}^{\text {perf }}$ is $\boldsymbol{\Pi}_{3}^{0}$-hard. Let $B$ be any $\boldsymbol{\Pi}_{3}^{0}$ subset of some zero-dimensional Polish space $Z$, and write $B=\bigcap_{n} A_{n}$, where the $A_{n}$ 's are $\boldsymbol{\Sigma}_{2}^{0}$. Let $\left(V_{n}\right)$ be a sequence of disjoint open subsets of $X$ accumulating to some point $a \in X$ (such a sequence exists because the denseness of $\mathcal{I}$ and $\mathcal{I}^{\text {perf }}$ implies that $X$ is perfect), and for each $n$, let $\Psi_{n}: Z \rightarrow \mathcal{K}\left(V_{n}\right)$ be a continuous map such that $\Psi_{n}^{-1}\left(\mathcal{I}^{\text {perf }}\right)=A_{n}$. Then the map $\Psi: Z \rightarrow \mathcal{K}(X)$ defined by $\Psi(z)=\{a\} \cup \bigcup_{n} \Psi_{n}(z)$ is continuous, and $\Psi(z)$ is $\mathcal{I}$-perfect if and only if all $\Psi_{n}(z)$ 's are; in other words, $\Psi^{-1}\left(\mathcal{I}^{\text {perf }}\right)=B$. This concludes the proof.

COROLlary 5.2. In the following cases, the family $\mathcal{I}^{\text {perf }}$ is a true $\mathbf{\Pi}_{3}^{0}$ subset of $\mathcal{K}(X)$ :

(1) $X$ is a perfect Polish space, and $\mathcal{I}=\{K \in \mathcal{K}(X) ; \lambda(K)=0\}$, where $\lambda$ is a Borel probability measure on $X$ with support $X$.

(2) $X$ is a perfect locally compact Polish space, and $\mathcal{I}$ is the family of nowhere dense compact sets.

(3) $X=E$ is a closed $\mathcal{M}_{0}$-set in some nondiscrete, second countable $L C A$ group $\mathbf{G}$, and $\mathcal{I}$ is the family of compact $\mathcal{U}_{0}$-sets contained in $X$.

Proof. In case (1), the set $\mathcal{I}$ is $G_{\delta}$, so $\mathcal{I}^{\text {perf }}$ is $\Pi_{3}^{0}$. Moreover, $\mathcal{I}$ is dense in $\mathcal{K}(X)$ (hence comeager) because $X$ is perfect, and $\mathcal{I}^{\text {perf }}$ is dense as well because $\lambda$ has support $X$. So $\mathcal{I}^{\text {perf }}$ is a true $\Pi_{3}^{0}$ set by Proposition 5.1. Case (2) is treated in the same way.

In case $(3), \mathcal{I}$ has the Baire property since it is $\boldsymbol{\Pi}_{1}^{1}$, and $\mathcal{I}^{\text {perf }}$ is $\boldsymbol{\Pi}_{3}^{0}$ because the $\sigma$-ideal $\mathcal{I}$ has a $\boldsymbol{\Sigma}_{3}^{0}$ hereditary basis (see [DStR], [KL], [M2] or $[\mathrm{T}]$ ); and Proposition 5.1 applies to any closed set $\widetilde{E} \in \mathcal{I}^{\text {perf }}$ contained in $E$, because $\mathcal{I}$ contains the family of Dirichlet sets, which is $G_{\delta}$ and contains all finite sets, so $\mathcal{I} \cap \mathcal{K}(\widetilde{E})$ is comeager in $\mathcal{K}(\widetilde{E})$.

REMARK. Examples (1) and (2) are given in [BD]. Case (3) was obtained in [M2], with a much more complicated proof.

Our last result concerns families of probability measures.

Proposition 5.3. Let $X$ be a Polish space, and let $\mathbf{B} \subset \mathbf{P}(X)$ be hereditary and closed under infinite convex combinations. Assume that $X$ is the support of some measure in $\mathbf{B}$, and that the polar $\sigma$-ideal $\mathcal{I}_{\mathbf{B}}=\{K \in$ $\mathcal{K}(X) ; \mu(K)=0$ for all $\mu \in \mathbf{B}\}$ is nonmeager in $\mathcal{K}(X)$. Then $\mathbf{B}$ is not $\boldsymbol{\Sigma}_{3}^{0}$.

Proof. Of course, we may assume that $\mathbf{B}$ is Borel. Moreover, it is in fact enough to show that $\mathbf{B}$ is not $\boldsymbol{\Pi}_{2}^{0}$ : indeed, since $\mathbf{B}$ is closed under 
infinite convex combinations, an argument similar to that used in the proof of Proposition 5.1 will then yield that $\mathbf{B}$ is $\mathbf{\Pi}_{3}^{0}$-hard; see [D] for the details. Now, $\mathcal{I}_{\mathbf{B}}$ is $\boldsymbol{\Pi}_{1}^{1}$ in $\mathcal{K}(X)$ because $\mathbf{B}$ is assumed to be Borel (hence $\boldsymbol{\Sigma}_{1}^{1}$ ). By Proposition 4.12 , it follows that $\mathbf{B}$ cannot be $\boldsymbol{\Pi}_{2}^{0}$.

Corollary 5.4. Let $X$ be a perfect Polish space, and let $\lambda$ be a Borel probability measure on $X$ with support $X$. Then $\mathbf{B}_{\lambda}=L^{1}(\lambda) \cap \mathbf{P}(X)$ is a true $\boldsymbol{\Pi}_{3}^{0}$ subset of $\mathbf{P}(X)$.

Proof. It was observed in Section 3 that $\mathbf{B}_{\lambda}$ is $\boldsymbol{\Pi}_{3}^{0}$. That $\mathbf{B}_{\lambda}$ is not $\boldsymbol{\Sigma}_{3}^{0}$ follows at once from 5.3, but it can also be proved directly as follows. Since $\lambda$ has support $X$, the family $\mathbf{B}_{\lambda}$ is dense in $\mathbf{P}(X)$. But $\mathbf{B}_{\lambda}$ is also meager in $\mathbf{P}(X)$, because it is disjoint from the dense $G_{\delta}$ set $\mathbf{P}(G)$, where $G$ is any $\lambda$-negligible dense $G_{\delta}$ subset of $X$; such a set $G$ exists because $X$ is perfect. By the Baire Category Theorem, $\mathbf{B}_{\lambda}$ cannot be $\boldsymbol{\Pi}_{2}^{0}$, and the conclusion easily follows.

Corollary 5.5. If $\mathbf{G}$ is a nondiscrete, second countable LCA group, then the family of all Rajchman probability measures on $\mathbf{G}$ is a true $\mathbf{\Pi}_{3}^{0}$ set.

Proof. This follows from 5.3 because the $\sigma$-ideal of all compact $\mathcal{U}_{0^{-}}$ subsets of $\mathbf{G}$ is comeager in $\mathcal{K}(\mathbf{G})$.

\section{References}

[BD] M. Balcerzak and U. B. Darji, Some examples of true $F_{\sigma \delta}$ sets, Colloq. Math. 86 (2000), 203-207.

[D] G. Debs, Polar $\sigma$-ideals of compact sets, Trans. Amer. Math. Soc. 347 (1995), 317-338.

[DStR] G. Debs et J. Saint-Raymond, Ensembles boréliens d'unicité et d'unicité au sens large, Ann. Inst. Fourier (Grenoble) 37 (1987), no. 3, 217-239.

[K1] A. S. Kechris, Classical Descriptive Set Theory, Grad. Texts in Math. 156, Springer, 1995.

[K2] - Hereditary properties of the class of closed sets of uniqueness for trigonometric series, Israel J. Math. 73 (1991), 189-198.

[KL] A. S. Kechris and A. Louveau, Covering theorems for uniqueness and extended uniqueness sets, Colloq. Math. 59 (1990), 63-79.

[Kö] T. W. Körner, The behavior of power series on their circle of convergence, in: Lecture Notes in Math. 995, Springer, 1983, 56-94.

[LP] L. A. Lindahl and F. Poulsen, Thin Sets in Harmonic Analysis, Lecture Notes in Pure and Appl. Math. 2, Marcel Dekker, 1971.

[M1] É. Matheron, How to recognize a true $\boldsymbol{\Sigma}_{3}^{0}$ set, Fund. Math. 158 (1998), 181-194.

[M2] - Sigma-idéaux polaires et ensembles d'unicité dans les groupes abéliens localement compacts, Ann. Inst. Fourier (Grenoble) 46 (1996), no. 2, 493-533.

[My] J. Mycielski, Independent sets in topological algebras, Fund. Math. 55 (1964), 139-147.

[R] W. Rudin, Fourier-Stieltjes transforms of measures on independent sets, Bull. Amer. Math. Soc. 66 (1960), 81-83. 
[S] S. Solecki, Covering analytic sets by families of closed sets, J. Symbolic Logic 59 (1994), 1022-1031.

[T] V. Tardivel, Fermés d'unicité dans les groupes abéliens localement compacts, Studia Math. 91 (1988), 1-15.

Université Bordeaux 1

351 cours de la Libération

33405 Talence Cedex, France

E-mail: Etienne.Matheron@math.u-bordeaux1.fr
Charles University Faculty of Mathematics and Physics Sokolovská 83

186 75, Praha 8, Czech Republic E-mail: zeleny@karlin.mff.cuni.cz

Received 29 March 2004;

in revised form 31 January 2005 